\title{
Do Alzheimer's Disease Patients Appear Younger than Their Real Age?
}

\author{
Zeynep Tufekcioglua Başar Bilgic ${ }^{\mathrm{b}}$ Abdullah Emir Zeylan ${ }^{\mathrm{c}}$ Albert Ali Salah ${ }^{\mathrm{c}}$ \\ Hamdi Dibeklioglud ${ }^{d}$ Murat Emre $^{b}$ \\ ${ }^{a}$ Department of Neurology, Faculty of Medicine, Istanbul Aydin University, Istanbul, Turkey; ${ }^{b}$ Department of \\ Neurology, Movement Disorders and Behavioural Neurology Unit, Istanbul Faculty of Medicine, \\ Istanbul University, Istanbul, Turkey; ' Department of Information and Computing Sciences, Utrecht University, \\ Utrecht, The Netherlands; ${ }^{d}$ Department of Computer Engineering, Bilkent University, Ankara, Turkey
}

\section{Keywords}

Alzheimer's disease $\cdot$ Age $\cdot$ Physical appearance

\begin{abstract}
Introduction: The most prominent risk factor of Alzheimer's disease (AD) is aging. Aging also influences the physical appearance. Our clinical experience suggests that patients with $A D$ may appear younger than their actual age. Based on this empirical observation, we set forth to test the hypothesis with human and computer-based estimation systems. Method: We compared 50 early-stage AD patients with 50 age and sex-matched controls. Facial images of all subjects were recorded using a video camera with high resolution, frontal view, and clear lighting. Subjects were recorded during natural conversations while performing Mini-Mental State Examination, including spontaneous smiles in addition to static images. The images were used for age estimation by 2 methods: (1) computer-based age estimation; (2) human-based age estimation. Computer-based system used a state-of-the-art deep convolutional neural network classifier to process the facial images contained in a single-video session and performed frame-based age estimation. Individuals who estimated the age by visual inspection of video sequences were
\end{abstract}

karger@karger.com

(c) 2020 S. Karger AG, Basel

www.karger.com/dem

Karger! chosen following a pilot selection phase. The mean error (ME) of estimations was the main end point of this study. Results: There was no statistically significant difference between the ME scores for AD patients and healthy controls $(p=0.33)$; however, the difference was in favor of younger estimation of the AD group. The average ME score for AD patients was lower than that for healthy controls in computer-based estimation system, indicating that AD patients were on average estimated to be younger than their actual age as compared to controls. This difference was statistically significant ( $p=$ 0.007). Conclusion: There was a tendency for humans to estimate $A D$ patients younger, and computer-based estimations showed that $A D$ patients were estimated to be younger than their real age as compared to controls. The underlying mechanisms for this observation are unclear.

(c) 2020 S. Karger AG, Basel

\section{Introduction}

Alzheimer's disease (AD) is the most common neurodegenerative disorder in the elderly. The most prominent risk factor for $\mathrm{AD}$ is aging. Aging also influences the physical appearance of an individual, including facial features, 
which in turn determine the perceived age of a person. Our clinical experience over the years suggests that patients with $\mathrm{AD}$ may appear younger than their actual age. Based on this empirical observation and without an a priori hypothesis on potential causes, we set forth to test the hypothesis if $\mathrm{AD}$ patients look younger than their chronological age as compared to their peers. In order to test this hypothesis, we compared the estimated age of $\mathrm{AD}$ patients and age and sex-matched healthy subjects using both computerized methods as well as age estimation by humans.

\section{Methods}

\section{Participants}

Fifty early-stage AD patients were consecutively recruited at Istanbul Faculty of Medicine, Department of Neurology, Behavioral Neurology Outpatient Clinic. The eligibility criteria for inclusion in the study were: (1) age $\geq 64$ years, (2) diagnosis of AD according to NINCDS-ADRDA criteria for probable AD [1], (3) clinical dementia rating scale score 0.5 or 1 [2]. As controls, 50 age- and sex-matched healthy individuals were recruited, among the family members of patients as well as volunteers among relatives of patients attending the general outpatient clinic. Inclusion criteria for healthy controls were: (1) Mini-Mental State Examination (MMSE) score $\geq 26$ [3], (2) no subjective or reported cognitive impairment, (3) no history of systemic, psychiatric, or neurological disorder. Exclusion criteria for both patients and healthy controls were: (1) severe visual impairment or hearing loss, (2) history of facial botulinum toxin injection, (3) having any major facial scar, (4) history of facial palsy, (5) using antipsychotic medication, (6) having parkinsonism or significant apathy in the neurological examination, and (7) geriatric depression scale score $>13[4]$.

The study was approved by the Ethical Committee of İstanbul Faculty of Medicine. All subjects and/or their next-of kin (in case of patients) provided written informed consent for the anonymous use of their data and images for age estimation.

\section{Facial Image Recordings}

Facial images of all subjects were recorded using a video camera with high resolution, frontal view, and clear lighting. As computerbased facial age estimation also leverages facial dynamics during facial expressions, subjects were recorded during natural conversations while performing MMSE, including spontaneous smiles in addition to static images. Video recordings consisted of RGB videos, recorded in $1,920 \times 1,080$ pixels at a rate of 25 frames per second. During recordings, none of the subjects used eyeglasses to avoid interference with computer processing and influence on age perception.

\section{Age Estimation}

The images were used for age estimation by 2 methods: (1) computer-based age estimation, (2) human-based age estimation.

\section{Computer-Based Age Estimation}

Computer-based system used a state-of-the-art deep convolutional neural network classifier to process the facial images contained in a single-video session and performed frame-based age estimation. For a single video, this produces around 2,000 frames with estimated ages. We fit a curve to all estimations of the session to predict a single age for the subject, which produces a better result compared to taking an average for non-Gaussian distributed predictions [5]. An established automatic facial estimation database and protocols called FG-NET were used to verify the accuracy of the system. Due to low quality, 6 videos of healthy subjects were excluded, and in total 94 videos were analyzed.

\section{Human-Based Age Estimation}

Individuals who estimated the age by visual inspection of video sequences were chosen following a pilot-selection phase as follows: among the employees of the Neurology Department, 15 volunteers were asked to estimate the age of randomly selected unfamiliar faces. The volunteers included 5 individuals from the age-group of $30-39,5$ from the age-group of 40-49, and 5 from the age-group of 50-59. They were shown video recordings of 31 individuals (including 11 healthy controls and 20 Alzheimer's patients) of various ages and were asked to estimate their ages. In each of the 3 agegroups, the individual with the lowest mean absolute error (MAE) score (see below) was identified, and these 3 individuals were defined as "estimators." They were then shown the video recordings of $\mathrm{AD}$ patients and healthy controls and asked to estimate their age. All 3 estimators used the same computer and standard screen adjustments for viewing the recordings (13.3-inch LED-backlit display with IPS technology). They were not aware of the diagnosis, and the videos of $\mathrm{AD}$ patients and healthy controls were shown in a mixed, random order.

MAE score was used to evaluate the accuracy of age estimation and to select the best estimators. Absolute error was defined as the difference in years between the estimated age and the real age of a subject. As shown below, MAE was calculated by adding absolute errors for all subjects without taking into account if a given estimation was higher or lower than the actual age. The total number in years was then divided by the number of subjects. Lower scores of MAE indicate more accurate estimations.

Mean error (ME) was the main end point of this study and calculated as follows: the errors in estimation for all subjects in a group were added together taking in account if the estimation was higher or lower than the actual age (whereby negative and positive values counteract each other) and then dividing the total number by the number of subjects in that group. Negative values of ME indicate that on average, subjects in that group were estimated to be younger than their actual age whereas positive values indicate that they were estimated to be older than their actual age. ME can be computed as follows:

$(\text { estimated age }- \text { actual age })_{n=1}+\ldots+($ estimated age - actual age $)$ total number of subjects

\section{Statistics}

Statistical analyses were performed using SPSS software version 22. Descriptive statistics were used to evaluate the demographics of the sample population, and mean age and sex distribution were tested for statistically significant differences. Kolmogorov-Smirnov/Shapiro-Wilk test was applied to analyze if the 
Table 1. Demographic and clinical variables of patients and control subjects

\begin{tabular}{lccc}
\hline & $\begin{array}{l}\mathrm{AD} \\
(n=50)\end{array}$ & $\begin{array}{l}\text { Healthy controls } \\
(n=50)\end{array}$ & $p$ value \\
\hline Age: mean, range (SD) & $74.30,64-87(5.92)$ & $72.84,65-85(5.81)$ & $0.17^{\mathrm{a}}$ \\
Gender: male, $n(\%)$ & $27(54)$ & $27(54)$ & $1.0^{\mathrm{b}}$ \\
MMSE score: mean, range (SD) & $23.54,12-30(4.26)$ & $28.84,24-30(1.31)$ & $<0.05^{\mathrm{c}}$ \\
CDR: mean, range (SD) & $0.71,0.5-1(0.25)$ & $0(0)$ & $<0.05^{\mathrm{b}}$ \\
\hline
\end{tabular}

CDR, clinical dementia rating score; MMSE, Mini-Mental State Examination; SD, standard deviation; AD, Alzheimer's disease. ${ }^{a}$ Mann-Whitney U test was used to compare variables between groups. ${ }^{b} \chi^{2}$ test was used to compare variables between groups. ${ }^{\mathrm{c}} t$ test was used to compare variables between groups.

Table 2. ME values of the human estimators and computer-based estimation

\begin{tabular}{|c|c|c|c|}
\hline & $\begin{array}{l}\mathrm{AD} \\
(n=50)\end{array}$ & $\begin{array}{l}\text { Healthy control } \\
(n=50)\end{array}$ & $p$ value \\
\hline Estimator 1, ME (SD) & $1.68(6.76)$ & $3.36(6.95)$ & $0.32^{\mathrm{a}}$ \\
\hline Estimator 2, ME (SD) & $-1.76(6.21)$ & $-1.26(4.96)$ & $0.79^{\mathrm{a}}$ \\
\hline Estimator 3, ME (SD) & $-0.56(7.90)$ & $2.08(5.93)$ & $0.08^{\mathrm{a}}$ \\
\hline \multirow[t]{2}{*}{ Mean value of the 3 estimators*, ME (SD) } & $-0.21(5.89)$ & $1.39(5.10)$ & $0.33^{\mathrm{a}}$ \\
\hline & $\begin{array}{l}\mathrm{AD} \\
(n=50)\end{array}$ & $\begin{array}{l}\text { Healthy control } \\
(n=44)\end{array}$ & $p$ value \\
\hline Mean value of the computer-based age estimation, ME & -9.7 & -4.48 & $0.007^{\mathrm{b}}$ \\
\hline
\end{tabular}

$\mathrm{AD}$, Alzheimer's disease; ME, mean error; SD, standard deviation. ${ }^{\text {a }}$ The Mann-Whitney U test was used to compare variables between groups. ${ }^{b} t$ test was used to compare variables between groups. ${ }^{*}$ Mean ME was calculated by pooling together error in estimations of all 3 estimators.

variables were normally distributed. There were 50 patients and 50 healthy subjects included in the human-based age estimation analysis. Age, MMSE, CDR scores, and ME values did not show normal distribution, so nonparametric Mann-Whitney $U$ test and $\chi^{2}$ test were used to compare the groups. In the computer-based estimation analysis, the number of the subjects was different (50 AD patients and 44 healthy subjects) data showing normal distribution, and parametric $t$ test was conducted for comparison of the ME values of the groups.

\section{Results}

Demographic and clinical variables are summarized in Table 1. Age range, mean age, and gender distribution were comparable between the patient and control subjects $(p=0.17$ and $p=1.0$, respectively). The mean MiniMental State Examination score was $23.54 \pm 4.26$ in the $\mathrm{AD}$ and 28.84 in the control group.

Do Alzheimer's Disease Patients Appear Younger?

\section{Human-Based Age Estimations}

Figure 1 shows the actual and estimated ages of all subjects by the "estimators" and Table 2 shows the ME values for human-based and computer-based estimations. There was no statistically significant difference between the ME scores for AD patients and healthy controls (Table 2). Even though statistically not significant, there was a difference between the ME values of $\mathrm{AD}$ patients and control subjects in favor of younger estimation of the AD group. Difference between the ME values of $\mathrm{AD}$ versus control subjects for the 3 estimators was $1.68,0.50$, and 2.64 years, all in favor of the AD group.

\section{Computer-Based Age Estimation}

The ME values of the computer-based estimations are shown in Table 2. The average ME score for AD patients was 5.22 lower than that for healthy controls indi- 


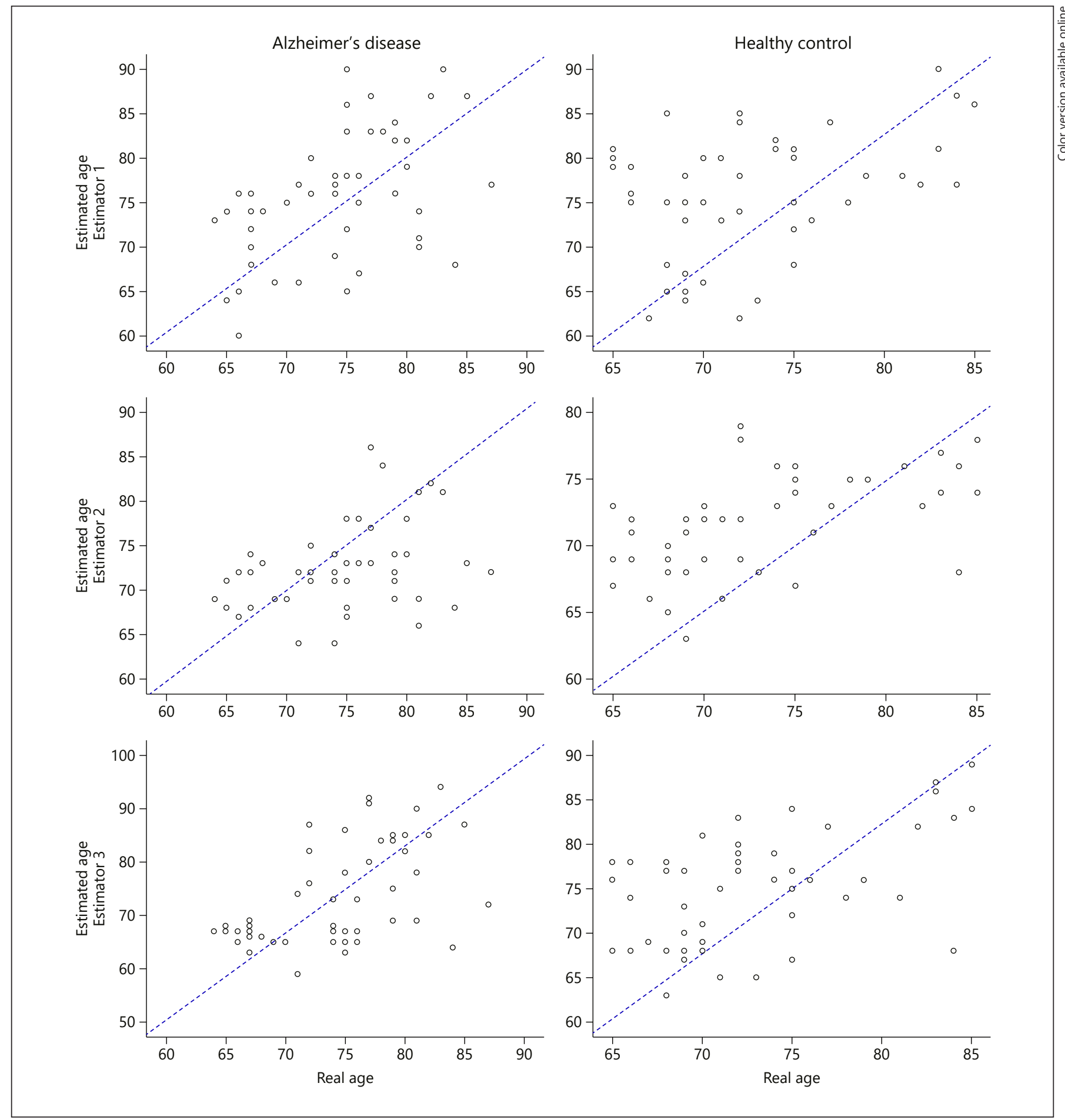

Fig. 1. The actual and estimated ages of all subjects with human-based estimation. Each dot represents 1 subject. Dots below the blue line represent cases in which the estimated age was lower than the actual, and dots above the blue line represent cases in which the estimated age was higher than the actual age. AD, Alzheimer's disease. 
cating that $\mathrm{AD}$ patients were on average estimated to be younger than their actual age as compared to controls. This difference was statistically significant $(p=0.007)$.

\section{Discussion}

In this study, there was a tendency for the ages of both healthy and $\mathrm{AD}$ subjects estimated to be younger than their actual age in computer-based estimations. Although statistically not significant, there was a tendency for humans to estimate $\mathrm{AD}$ patients younger than their actual age as compared to controls. Computerbased estimations showed that ages of $\mathrm{AD}$ patients were estimated to be significantly younger than the control subjects.

Factors underlying for a younger estimation of $\mathrm{AD}$ patients are unclear. Appearance of age is closely related to the physical changes which emerge with aging. Health status and environmental factors such as, sun exposure, smoking, BMI, social class, and marital status may all influence the perceived age $[6,7]$. Wrinkles and white hair are obvious influences on the estimated age, but additional factors such as facial expressions may also influence our estimations. AD pathology initially affects the limbic areas of the brain, which are highly associated with memory functions and emotions. They have connections with the nucleus of facial nerve, which innervates facial mimic muscles, and they have interconnections with cortical and subcortical areas, which were thought to be involved in the generation of emotional facial expressions [8]. AD patients were found to have altered facial mimic activity and expressions of emotions during emotional states [9]. Since facial mimics and expressions are one of the important clues used in estimating ages, altered facial mimics in AD patients may be the reason for their relatively younger appearance.

There are several caveats to our findings. We recruited patients older than 65 years at earlier stages of the disease. We also excluded patients with depression. We have done so in order to obtain a homogenous population typical of $\mathrm{AD}$ and also to exclude changes in facial expression associated with apathy in the later stages of the disease or due to depression. These limitations may render our results not generalizable in all cases.

An open question is if the static properties, facial dynamics, or both of these influenced the younger appearance of AD patients in the computer-based analysis. In recent years, many computer-based approaches have

Do Alzheimer's Disease Patients Appear Younger? been developed for age estimation for different purposes, such as forensics [10]. Most of the automatic facial age estimation approaches use static features. Recently, dynamic features were also introduced, arguing that aging changes the muscle tone in the face. Facial dynamics are also affected by morphological changes such as, muscle loss, fat tissue, and cartilage growth. Automatic facial age estimation studies established that facial dynamics can provide additional cues about the age of a person [11]. Fusing facial dynamics with static appearance features may enhance age estimation.

In conclusion, in this study, $\mathrm{AD}$ patients were estimated to look younger than their actual age as well as compared to their age-matched healthy controls, in particular by computer-based estimations. The difference is, however small, that these results must be interpreted cautiously and need confirmation. If confirmed in other studies, a mismatch between chronological and facial age may be an indicator of Alzheimer's disease. In order to assess if this is a phenomenon inherent to dementia of any cause, future studies may evaluate patients with other forms of degenerative dementias.

\section{Acknowledgements}

We thank the patients and healthy subjects who participated in our study.

\section{Statement of Ethics}

All the procedures performed in this study were in accordance with ethical standards laid down in 1964 Declaration of Helsinki and its later amendments or comparable ethical standards. All patients gave their written informed consent before study enrollment.

\section{Conflict of Interest Statement}

The authors declare that they have no conflict of interest to declare.

\section{Funding Sources}

The authors have no relevant financial or nonfinancial relationships to disclose. 


\section{Author Contributions}

All authors contributed to the study conception and design. Material preparation was performed by Zeynep Tufekcioglu, Basar Bilgic, and Albert Ali Salah. Data collection and analysis were performed by all authors. Zeynep Tufekcioglu, Basar Bilgic, Abdullah
Emir Zeylan, and Albert Ali Salah did the statistical analyses. All authors contributed to interpretation of the results. The first draft of the manuscript was written by Zeynep Tufekcioglu. Review and supervision were performed by Basar Bilgic and Murat Emre. All authors commented on previous versions of the manuscript and read and approved the final manuscript.

\section{References}

1 McKhann G, Drachman D, Folstein M, Katzman R, Price D, Stadlan EM. Clinical diagnosis of Alzheimer's disease: report of the NINCDS-ADRDA Work Group under the auspices of department of health and human services task force on Alzheimer's disease. Neurology. 1984 Jul;34(7):939-44.

2 Hughes CP, Berg L, Danziger WL, Coben LA, Martin RL. A new clinical scale for the staging of dementia. Br J Psychiatry. 1982 Jun; 140: $566-72$.

3 Folstein MF, Folstein SE, McHugh PR. Minimental state. A practical method for grading the cognitive state of patients for the clinician. J Psychiatr Res. 1975 Nov;12(3):189-98.

4 Brink TL, Yesavage JA, Lum O, Heersema $\mathrm{PH}$, Adey M, Rose TL. Screening tests for geriatric depression. Clinical Gerontologist. 1982;1(1):37-43.
5 Zeylan AE, Salah AA, Dibeklioğlu H, Tüfekçioğlu Z, Bilgiç B, Emre M. Do Alzheimer's patients appear younger than their age? a study with automatic facial age estimation. Ninth international conference on image processing theory, tools and applications (IPTA). Turkey, Istanbul; 2019. p. 1-6.

6 Mayes AE, Murray PG, Gunn DA, Tomlin CC, Catt SD, Wen YB, et al. Environmental and lifestyle factors associated with perceived facial age in Chinese women. PLoS One. 2010 Dec 13;5(12):e15270.

7 Rexbye H, Petersen I, Johansens M, Klitkou L, Jeune B, Christensen K. Influence of environmental factors on facial ageing. Age Ageing. 2006 Mar;35(2):110-5.
8 Tucker DM, Derryberry D, Luu P. Anatomy and physiology of human emotion: vertical integration of brainstem, limbic, and cortical systems. In: Borod JC, editor. The neuropsychology of emotion. New York: Oxford University Press; 2000. p. 56-79.

9 Burton KW, Kaszniak AW. Emotional experience and facial expression in Alzheimer's disease. Neuropsychol Dev Cogn B Aging Neuropsychol Cogn. 2006 Sep-Dec;13(3-4): 636-51.

10 Albert AM, Ricanek K Jr, Patterson E. A review of the literature on the aging adult skull and face: implications for forensic science research and applications. Forensic Sci Int. 2007 Oct 2;172(1):1-9.

11 Dibeklioglu H, Gevers T, Salah AA, Valenti R. A smile can reveal your age: enabling facial dynamics in age estimation. 20th ACM Int. Conf. on Multimedia, ACM; 2012. p. 209-18. 